\title{
Briefs
}

\section{A High-Performance Micromachined RF Monolithic Transformer With Optimized Pattern Ground Shields (OPGS) for UWB RFIC Applications}

Yo-Sheng Lin, Chi-Chen Chen, Hsiao-Bin Liang, Pei-Kang Tsai, Chang-Zhi Chen, Jin-Fa Chang, Tao Wang, and Shey-Shi Lu

\begin{abstract}
In this brief, we demonstrate that high-quality-factor and low-power-loss transformers can be obtained if the optimized pattern ground shields (OPGS) of polysilicon is adopted and the CMOS processcompatible backside inductively coupled-plasma (ICP) deep-trench technology is used to selectively remove the silicon underneath the transformers completely. OPGS means that the redundant PGS of a traditional complete PGS, which is right below the spiral metal lines of the transformer, is removed for the purpose of reducing the large parasitic capacitance. The results show that, if the OPGS was adopted and the backside ICP etching was done, a $69.3 \%$ and a $253.6 \%$ increase in quality factor, a $10.5 \%$ and a $14 \%$ increase in magnetic-coupling factor $\left(k_{\operatorname{Im}}\right)$, a $17.2 \%$ and a $51.1 \%$ increase in maximum available power gain $\left(G_{\mathrm{A} \max }\right)$, and a 0.682 - and a 1.79-dB reduction in minimum noise factor $\left(\mathrm{NF}_{\min }\right)$ were achieved at 5 and $8 \mathrm{GHz}$, respectively, for a bifilar transformer with an overall dimension of $230 \times 215 \boldsymbol{\mu m}^{2}$.
\end{abstract}

Index Terms-Micromachined, optimized pattern ground shield (OPGS), power gain, quality-factor, radio frequency integrated circuits (RFICs), transformer.

\section{INTRODUCTION}

The minimum noise figure $\mathrm{NF}_{\min }$ of a transformer (i.e., $10 \cdot \log (1 /$ $\left.G_{\mathrm{A} \max }\right)$, in which $G_{\mathrm{A} \max }$ is the maximum available power gain [1], is a direct index of the power loss (in decibels) of the device. In the design of low-voltage and high-performance CMOS (or BiCMOS) radio frequency integrated circuits (RFICs) [2]-[4], the quality factor ( $Q$-factor) and $\mathrm{NF}_{\text {min }}$ performances of the transformers used are crucial for the phase-noise performance of the voltage-controlled oscillators and the noise figure performance of the low-noise amplifiers. However, the $Q$-factor and $\mathrm{NF}_{\text {min }}$ performances of $\mathrm{RF}$ transformers fabricated on normal silicon substrates are normally not satisfactory mainly due to their capacitive and electromagnetic coupling with the silicon substrate. Adding polysilicon (or metal) pattern ground shields (PGS) underneath the transformers (or inductors) has been widely adopted to suppress the electromagnetic-coupling-induced loss (or eddy-current loss) in the silicon substrate [5], [6], so the maximum $Q$-factor of the devices can be improved. However, the large parasitic capacitance between the PGS and the devices leads to poorer $Q$-factor in high-frequency and lower self-resonant frequency $\left(f_{\mathrm{SR}}\right)$. Fortunately, this problem can be improved by our proposed optimized PGS (OPGS). OPGS means that the redundant PGS of a traditional complete PGS, which is right below the spiral metal lines of an

Manuscript received August 21, 2006; revised November 27, 2006. The review of this brief was arranged by Editor K. Najafi.

Y.-S. Lin, C.-C. Chen, H.-B. Liang, P.-K. Tsai, C.-Z. Chen, and J.-F. Chang are with the Department of Electrical Engineering, National Chi Nan University, Puli 545, Taiwan, R.O.C.

T. Wang and S.-S. Lu are with the Graduate Institute of Electronics Engineering and Department of Electrical Engineering, National Taiwan University, Taipei 106, Taiwan, R.O.C. (e-mail address: sslu@ntu.edu.tw).

Color versions of one or more of the figures in this brief are available online at http://ieeexplore.ieee.org.

Digital Object Identifier 10.1109/TED.2006.890390

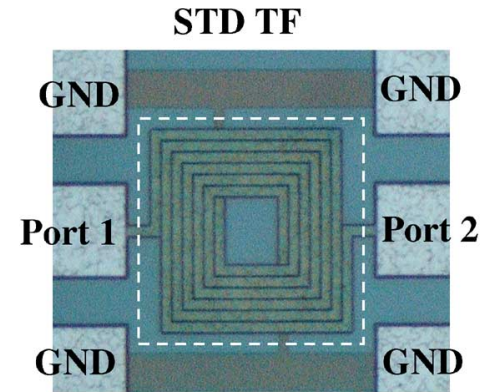

(a)

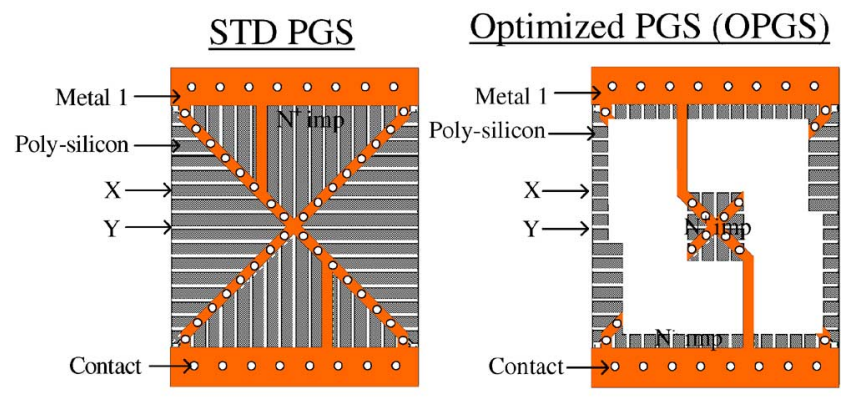

(b)

Fig. 1. (a) Top-view die photo of a bifilar transformer with metal width of $10 \mu \mathrm{m}$, coil spacing (between the primary and the secondary coil) of $2 \mu \mathrm{m}$, inner dimension of $62 \times 73 \mu \mathrm{m}$, and overall dimension of $230 \times 215 \mu \mathrm{m}$. (b) Schematic diagrams of the proposed OPGS formed by the $\mathrm{N}^{+}$polysilicon layer, and a corresponding traditional complete PGS (STD PGS) for comparison.

RF-passive device, is removed for the purpose of reducing the large parasitic capacitance [see Fig. 1(b)].

$\mathrm{NF}_{\min }$ (or $G_{\mathrm{A} \max }$ ) of a transformer is a function of its $Q$-factors $\left(\mathrm{Q}_{1}\right.$ and $\left.\mathrm{Q}_{2}\right)$, magnetic-coupling factor $\left(k_{\mathrm{Im}}\right)$, and resistive-coupling factor $\left(k_{\mathrm{Re}}\right)$ [6], [7]. In this brief, we demonstrate that the $\mathrm{NF}_{\min }$ (or $\left.G_{\mathrm{A} \text { max }}\right)$ performance of a transformer can be optimized if the combination of OPGS (mainly for improving $k_{\mathrm{Im}}$ ) and CMOS-compatible backside inductively coupled-plasma (ICP) deep-trench technology (mainly for improving $\mathrm{Q}_{1}$ and $\mathrm{Q}_{2}$ ) is adopted.

\section{TRANSFORMER AND OPGS STRUCTURE}

Fig. 1(a) shows the top-view die photo of a symmetrical bifilar transformer, which has turn numbers of three, metal width of $10 \mu \mathrm{m}$, coil spacing of $2 \mu \mathrm{m}$, inner dimension of $62 \times 73 \mu \mathrm{m}$, and overall dimension of $230 \times 215 \mu \mathrm{m}$. Port- 1 and Port- 2 stand for the input port and the output port of the network analyzer, respectively. The transformers under study were fabricated with a $0.35 \mu \mathrm{m} \mathrm{SiGe}$ BiCMOS technology on a p-type silicon substrate with thickness of $318 \mu \mathrm{m}$ and resistivity of $10 \Omega \cdot \mathrm{cm}$. The main features of the backend processes are as follows. There were three metal layers, named M1-M3 from bottom to top. The thickness of M3 was $3.05 \mu \mathrm{m}$, and that of M1 and M2 were $0.665 \mu \mathrm{m}$. The oxide thickness was $1 \mu \mathrm{m}$ between adjacent metal layers, and $1.1 \mu \mathrm{m}$ between M1 and the silicon substrate. Fig. 1(b) shows the schematic of the proposed OPGS formed by the $\mathrm{N}^{+}$polysilicon layer with thickness of $0.28 \mu \mathrm{m}$ and sheet resistance of $102 \pm 15.3 \Omega /$ sq. The width $(X)$ and slot $(Y)$ were 4 and 
Photolithography :

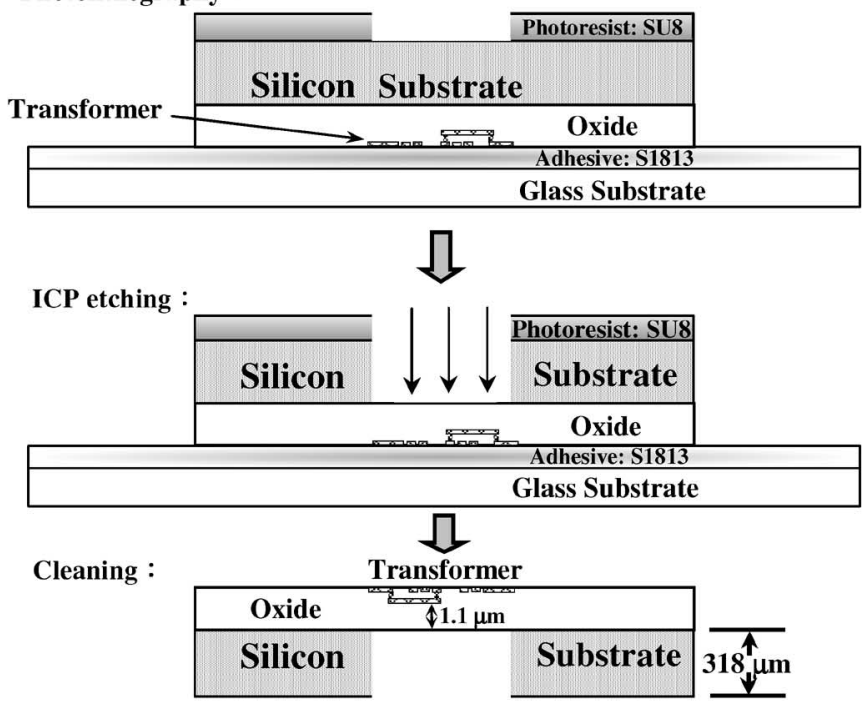

(a)

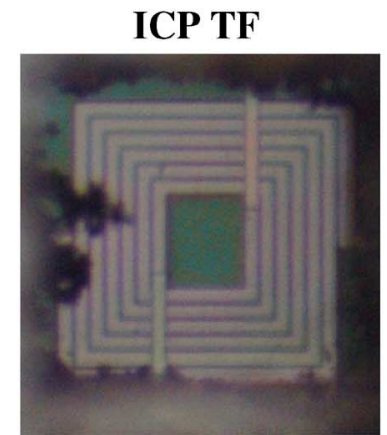

\section{OPGS-ICP TF}

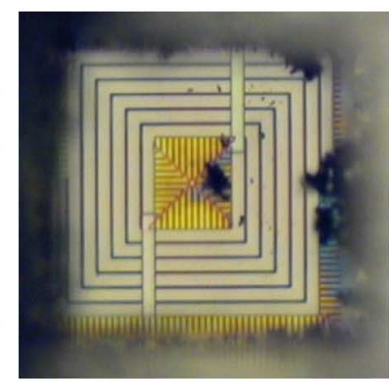

(b)

Fig. 2. (a) Process steps of the backside ICP deep-trench etching technology. (b) Backside die photos of the transformer without OPGS after the backside ICP etching (ICP TF), and the transformer with OPGS after the backside ICP etching (OPGS-ICP TF).

$1 \mu \mathrm{m}$, respectively. To minimize the voltage variation in the OPGS, an M1 pattern in the shape of a cross, which connects the polysilicon layer via contacts, had been added. What is also shown in Fig. 1(b) is the schematic of a corresponding traditional complete PGS (STD PGS) for comparison.

\section{DeEP-TREnCh TEChNOLOGY}

The processing steps of our CMOS-compatible backside ICP deep-trench technology are shown in Fig. 2(a) and described as follows. First, for the ease of chip handling, the front side of the die $(\sim 5 \times 5 \mathrm{~mm})$ with transformers was stuck to a glass substrate. Note that this process step can be omitted when applied in mass production, because the wafer diameter is large. After standard photolithography processes on the backside of the die, the ICP etching was used to remove the silicon underneath the transformers. Specifically, the silicon below the rectangular area surrounded by the dash line as indicated in Fig. 1(a) was fully dry-etched away. The main gases used during the ICP-etching process were alternate $\mathrm{SF}_{6}$ (for etching) and $\mathrm{C}_{4} \mathrm{~F}_{8}$ (for passivation) with a 17 -s cycle. Finally, the adhesive and photoresist, which covered the front-side and backside of the die, respectively, were removed for test purpose. Compared with the traditional backside wet bulk micromachining in [8], the dry ICP etching has the advantages of forming vertical sidewalls and being

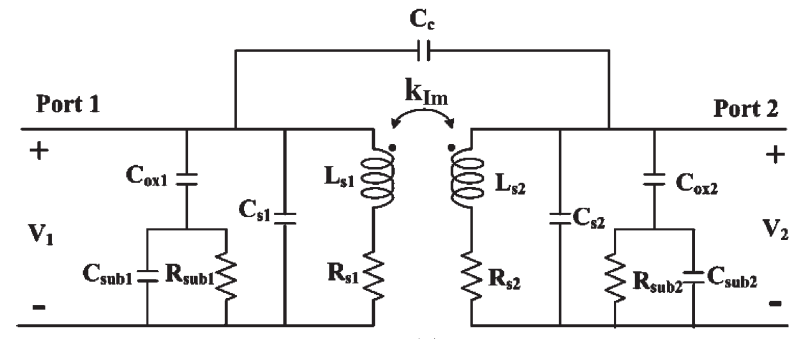

(a)

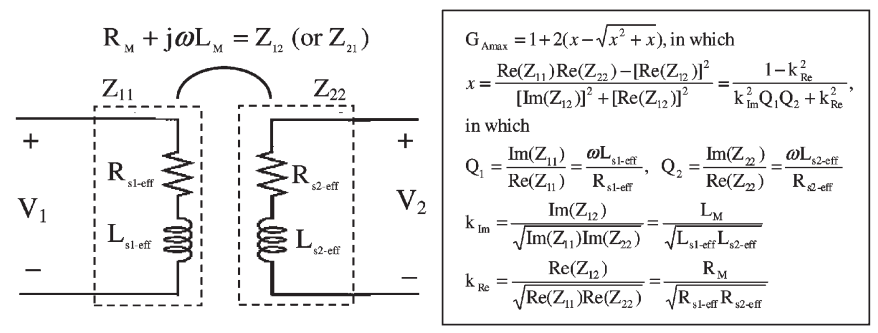

(b)

Fig. 3. Simplified small-signal equivalent-circuit model of a monolithic RF transformer. Expressions of $G_{\mathrm{A} \text { max }}, \mathrm{Q}_{1}, \mathrm{Q}_{2}, k_{\mathrm{Re}}$, and $k_{\mathrm{Im}}$ are also shown.

fully CMOS process compatible since it is a standard processing technique in modern CMOS technology.

The ICP-etching rate of silicon and oxide were about 2 and $0.02 \mu \mathrm{m} / \mathrm{min}$, respectively. That is, silicon to oxide ICP-etch selectivity was about 100 . Since the ratio between the silicon substrate thickness $(318 \mu \mathrm{m})$ and the oxide thickness underneath M1 layer $(1.1 \mu \mathrm{m})$ was high $(\approx 289.1)$, in this brief, we precisely control the etching time (159 $\mathrm{min}$ ) to fully remove silicon while keeping oxide underneath M1 layer nearly intact. Another feasible approach is to thin down the silicon substrate to about $100 \mu \mathrm{m}$ by mechanical lapping [9] before the ICP-etching postprocess. Since the ICP-etching time was shortened from 159 to $50 \mathrm{~min}$, the process margin (of keeping oxide underneath M1 layer nearly intact) has been improved.

Fig. 2(b) shows the backside die photos of the transformer without OPGS after the backside ICP etching (ICP TF) and the transformer with OPGS after the backside ICP etching (OPGS-ICP TF). The exposed front-side on-chip transformer and PGS were visible to the naked eye. This means the silicon underneath the transformers is indeed nearly fully dry-etched away.

\section{RESUlts AND Discussions}

The $S$-parameter measurements were performed from 0.1 to $20 \mathrm{GHz}$ by an HP-8510C vector network analyzer. Fig. 3(a) shows the complete small-signal equivalent-circuit model of a transformer on a silicon substrate [10], which can be simplified to the one shown in Fig. 3(b). Based on the simplified transformer model in Fig. 3(b), it is convenient to use the $Z$-parameters $Z_{\mathrm{ij}}(i, j=1,2)$ to characterize the electrical properties of a transformer [6]. $Z_{11}\left(=R_{\mathrm{s} 1-\mathrm{eff}}+\right.$ $\left.\mathrm{j} \omega L_{\mathrm{s} 1-\mathrm{eff}}\right)$ and $Z_{22}\left(=R_{\mathrm{s} 2-\text { eff }}+\mathrm{j} \omega L_{\mathrm{s} 2-\text { eff }}\right)$ represent the equivalent single-port (i.e., one port is short with the other coil open) input impedance of the primary and the secondary coil inductor, respectively, of the transformer. $Z_{12}=Z_{21}\left(=R_{M}+\mathrm{j} \omega L_{M}\right)$ represents the mutual impedance of the transformer. That is, $L_{\mathrm{s} 1-\mathrm{eff}}, L_{\mathrm{s} 2-\mathrm{eff}}$, and $L_{M}$ represent the primary, secondary, and mutual inductances, respectively, and $R_{\mathrm{s} 1-\mathrm{eff}}, R_{\mathrm{s} 2-\mathrm{eff}}$, and $R_{M}$ represent the primary, secondary, and mutual resistances, respectively, of the transformer. What is also shown in Fig. 3(b) are the expressions of the $G_{\mathrm{A} \text { max }}, Q$-factors of the primary coil $\left(\mathrm{Q}_{1}\right)$ and the secondary coil $\left(\mathrm{Q}_{2}\right), k_{\mathrm{Im}}$, and $k_{\mathrm{Re}}$ of a transformer 
TABLE I

Detailed Comparisons of the Extracted Parameters of a SET OF Bifilar Transformers With the SAME Layout BUt DifFerent PGS STRUCTURES

\begin{tabular}{|c|c|c|c|c|c|c|c|c|c|c|c|}
\hline $\begin{array}{c}\text { PGS } \\
\text { structure }\end{array}$ & $\begin{array}{c}\text { Transformer } \\
\text { structure }\end{array}$ & $\begin{array}{c}\text { Overall } \\
\text { dimension } \\
\left(\mu \mathrm{m}^{2}\right)\end{array}$ & $\begin{array}{c}\text { Inner } \\
\text { dimension } \\
\left(\mu \mathrm{m}^{2}\right)\end{array}$ & Technology & $\begin{array}{c}f_{\mathrm{SR}} \\
(\mathrm{GHz})\end{array}$ & $\begin{array}{c}\text { Frequency } \\
(\mathrm{GHz})\end{array}$ & $\mathrm{Q}_{1}$ & $\mathrm{Q}_{2}$ & $\mathrm{k}_{\mathrm{Im}}$ & $\mathrm{k}_{\mathrm{Re}}$ & $\mathrm{G}_{\mathrm{Amax}}$ \\
\hline \multirow{3}{*}{$\begin{array}{l}\text { No PGS } \\
\text { (STD TF) }\end{array}$} & \multirow{12}{*}{$\begin{array}{c}\text { Bifilar } \\
\text { (on } 318 \mu \mathrm{m}- \\
\text { thick normal } \\
\text { Si substrate) }\end{array}$} & \multirow{12}{*}{$230 \times 215$} & \multirow{12}{*}{$62 \times 73$} & \multirow{12}{*}{$\begin{array}{c}0.35 \mu \mathrm{m} \\
\text { SiGe } \\
\text { BiCMOS }\end{array}$} & \multirow{3}{*}{15.6} & 2.4 & 6.48 & 6.71 & 0.70 & 0.39 & 0.64 \\
\hline & & & & & & 4.2 & 6.12 & 7.14 & 0.69 & 0.49 & 0.67 \\
\hline & & & & & & 5.2 & 5.55 & 6.63 & 0.69 & 0.52 & 0.66 \\
\hline \multirow{3}{*}{$\begin{array}{l}\text { STD PGS } \\
\left(\mathrm{N}^{+} \text {imp.) }\right. \\
\text { (PGS TF) }\end{array}$} & & & & & \multirow{3}{*}{10.1} & 2.4 & 6.23 & 6.44 & 0.80 & 0.51 & 0.68 \\
\hline & & & & & & 4.2 & 6.94 & 7.77 & 0.82 & 0.61 & 0.75 \\
\hline & & & & & & 5.2 & 6.59 & 7.97 & 0.83 & 0.68 & 0.77 \\
\hline \multirow{3}{*}{$\begin{array}{c}\text { STD PGS } \\
\left(\mathrm{P}^{+} \text {imp. }\right)\end{array}$} & & & & & \multirow{3}{*}{10} & 2.4 & 6.30 & 6.41 & 0.80 & 0.51 & 0.68 \\
\hline & & & & & & 4.2 & 7.06 & 7.53 & 0.82 & 0.62 & 0.75 \\
\hline & & & & & & 5.2 & 6.75 & 7.42 & 0.84 & 0.69 & 0.77 \\
\hline \multirow{3}{*}{$\begin{array}{c}\text { OPGS } \\
\left(\mathrm{N}^{+} \text {imp.) }\right. \\
\text { (OPGS TF) }\end{array}$} & & & & & \multirow{3}{*}{11.1} & 2.4 & 7.85 & 7.92 & 0.80 & 0.42 & 0.72 \\
\hline & & & & & & 4.2 & 9.58 & 10.60 & 0.82 & 0.49 & 0.79 \\
\hline & & & & & & 5.2 & 8.64 & 10.10 & 0.83 & 0.60 & 0.80 \\
\hline
\end{tabular}

TABLE II

Extracted Small-Signal Equivalent-Circuit Parameters of STD TF (Without PGS), PGS TF (With STD PGS), OPGS TF (WITH OPGS), ICP TF (WITHOUT PGS), AND OPGS-ICP TF (WITH OPGS)

\begin{tabular}{|c|c|c|c|c|c|}
\hline Technology & \multicolumn{5}{|c|}{ TSMC $0.35 \mu \mathrm{m}$ SiGe BiCMOS } \\
\hline Transformer ID & $\begin{array}{c}\text { STD TF } \\
\text { (w/o PGS) }\end{array}$ & $\begin{array}{c}\text { PGS TF } \\
\text { (w/i STD PGS) }\end{array}$ & $\begin{array}{c}\text { OPGS TF } \\
\text { (w/i OPGS) }\end{array}$ & $\begin{array}{c}\text { ICP TF } \\
\text { (w/o PGS) }\end{array}$ & $\begin{array}{l}\text { OPGS-ICP TF } \\
\text { (w/i OPGS) }\end{array}$ \\
\hline Structure & \multicolumn{5}{|c|}{ Bifilar } \\
\hline Width $(\mu \mathrm{m})$ & \multicolumn{5}{|c|}{10} \\
\hline Spacing $(\mu \mathrm{m})$ & \multicolumn{5}{|c|}{2} \\
\hline Turn number $(\mathrm{N})$ & \multicolumn{5}{|c|}{3} \\
\hline Turn ratio & \multicolumn{5}{|c|}{$1: 1$} \\
\hline Premary coil & \multicolumn{5}{|c|}{ M6 } \\
\hline Secondary coil & \multicolumn{5}{|c|}{ M6 } \\
\hline Overall dimension $\left(\mu \mathrm{m}^{2}\right)$ & \multicolumn{5}{|c|}{$230 \times 215$} \\
\hline Inner dimension $\left(\mu \mathrm{m}^{2}\right)$ & \multicolumn{5}{|c|}{$62 \times 73$} \\
\hline Frequency range & $\mathrm{DC}-15.6 \mathrm{GHz}$ & \multicolumn{2}{|c|}{$\mathrm{DC}-11 \mathrm{GHz}$} & $\mathrm{DC}-15.6 \mathrm{GHz}$ & $\mathrm{DC}-11 \mathrm{GHz}$ \\
\hline $\mathrm{R}_{\mathrm{s} 1}(\Omega)$ & 1.97 & 2.01 & 1.99 & 1.97 & 1.99 \\
\hline $\mathrm{L}_{\mathrm{s} 1}(\mathrm{nH})$ & 1.85 & 1.81 & 1.83 & 1.85 & 1.83 \\
\hline $\mathrm{C}_{\mathrm{s} 1} \quad(\mathrm{fF})$ & 10 & 10 & 10 & 10 & 10 \\
\hline $\mathrm{R}_{\mathrm{s} 2}(\Omega)$ & 1.97 & 2.01 & 1.99 & 1.97 & 1.99 \\
\hline $\mathbf{L}_{\mathrm{s} 2} \quad(\mathrm{nH})$ & 1.85 & 1.81 & 1.83 & 1.85 & 1.83 \\
\hline $\mathrm{C}_{\mathrm{s} 2} \quad(\mathrm{fF})$ & 10 & 10 & 10 & 10 & 10 \\
\hline $\mathrm{C}_{\mathrm{ox} 1}(\mathrm{fF})$ & 220 & 3000 & 225 & 220 & 225 \\
\hline $\mathrm{C}_{\text {sub1 }}(\mathrm{fF})$ & 25 & 65 & 65 & 4 & 6 \\
\hline $\mathrm{R}_{\text {sub } 1}(\Omega)$ & 320 & 950 & 950 & 2400 & 2600 \\
\hline $\mathrm{C}_{\mathrm{Ox} 2}(\mathrm{fF})$ & 220 & 3000 & 225 & 220 & 225 \\
\hline $\mathrm{C}_{\mathrm{sub} 2}(\mathrm{fF})$ & 25 & 65 & 65 & 4 & 6 \\
\hline $\mathrm{R}_{\mathrm{sub} 2}(\Omega)$ & 320 & 950 & 950 & 2400 & 2600 \\
\hline $\mathrm{k}_{\mathrm{Im}}$ & 0.7 & 0.8 & 0.8 & 0.7 & 0.8 \\
\hline $\mathrm{C}_{\mathrm{c}}(\mathrm{fF})$ & 10 & 10 & 10 & 10 & 10 \\
\hline
\end{tabular}

in terms of its measured $Z$-parameters, which can be converted from the measured $S$-parameters.

Table I shows a detailed comparison of the extracted parameters of a set of bifilar transformers with the same layout but with different PGS structure. As can be seen, the transformer with OPGS is the best from the view points of $\mathrm{Q}_{1}, \mathrm{Q}_{2}, G_{\mathrm{A} \max }$, and $\mathrm{NF}_{\min }$. Besides, the effect of the STD PGS formed by the $\mathrm{N}^{+}$polysilicon layer (with thickness of $0.28 \mu \mathrm{m}$ and sheet resistance of $102 \pm 15.3 \Omega / \mathrm{sq}$ ) on the transformer is about the same as that of the STD PGS formed by the $\mathrm{P}^{+}$polysilicon layer (with thickness of $0.28 \mu \mathrm{m}$ and sheet resistance of $1035 \pm 155 \Omega / \mathrm{sq})$. The measured $G_{\mathrm{A} \max }(0.78$ at $5.2 \mathrm{GHz})$ of our OPGS-ICP transformer (overall dimension of $230 \times 215 \mu \mathrm{m}$ ) is better than that $(0.603$ at $5.2 \mathrm{GHz})$ of a transformer with dimension of $300 \times 300 \mu \mathrm{m}$ on a quartz substrate [6] and better than that $(0.764$ at $5.2 \mathrm{GHz}$ ) of a transformer with dimension of $280 \times 280 \mu \mathrm{m}$ on a porous silicon substrate [7]. 


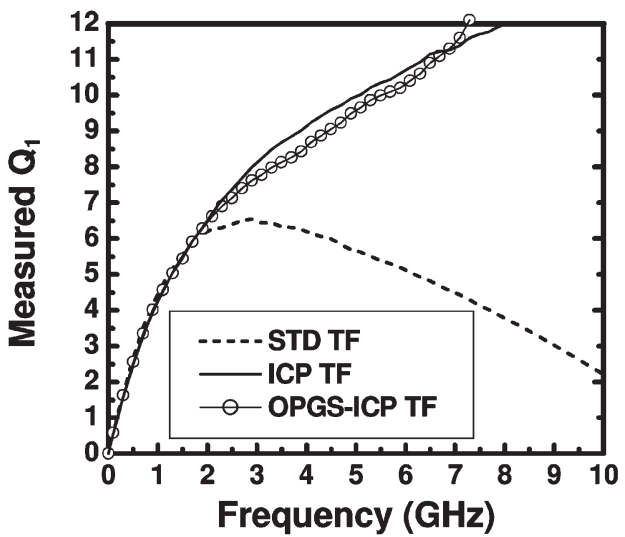

(a)

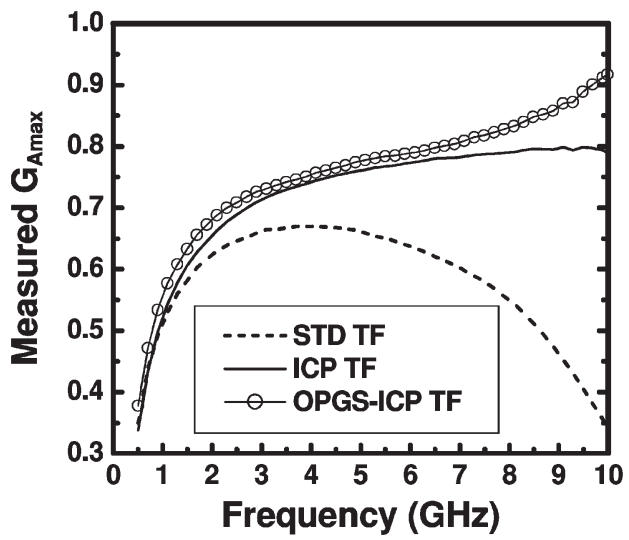

(c)

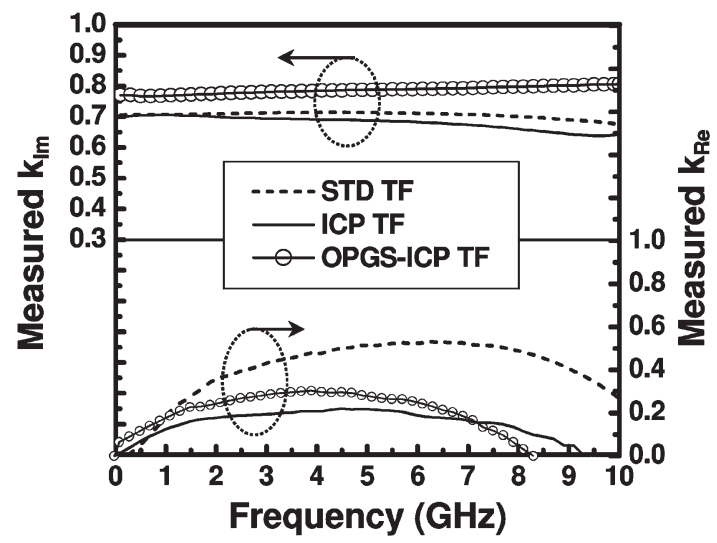

(b)

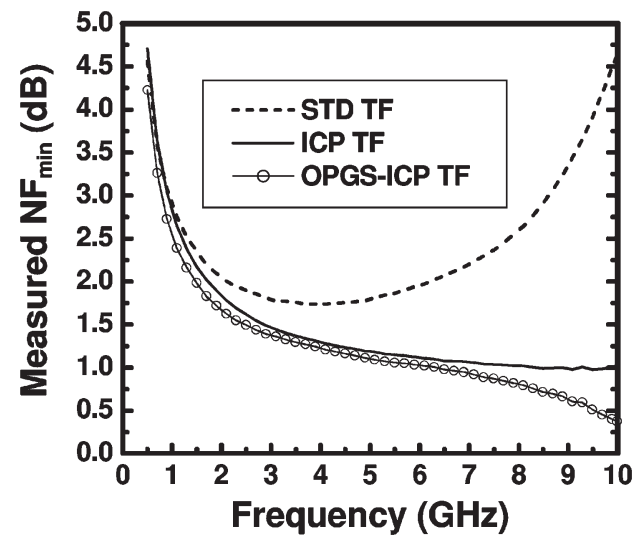

(d)

Fig. 4. Measured (a) $\mathrm{Q}_{1}$, (b) $k_{\mathrm{Im}}$ and $k_{\mathrm{Re}}$, (c) $G_{\mathrm{A} \max }$, and (d) $\mathrm{NF}_{\min }$ versus frequency characteristics of STD TF, ICP TF, and OPGS-ICP TF.

Table II shows a summary of the extracted small-signal equivalent circuit parameters of STD TF (without PGS), PGS TF (with STD PGS), OPGS TF (with OPGS), ICP TF (without PGS), and OPGSICP TF (with OPGS). The extracted values of $\mathrm{C}_{\mathrm{ox} 1}$ and $\mathrm{C}_{\mathrm{ox} 2}$ of the OPGS TF are lower than those of the PGS TF, since there is no polysilicon layer and gate-oxide layer directly beneath the spiral metal lines of the OPGS TF. This explains why in Table I that the OPGS TF exhibits better $\mathrm{Q}_{1}, \mathrm{Q}_{2}, f_{\mathrm{SR} 1}$, and $f_{\mathrm{SR} 2}$ performances than the PGS TF.

Fig. 4(a) shows the measured $Q_{1}$ versus frequency characteristics of STD TF (i.e., without both OPGS and ICP etching), ICP TF, and OPGS-ICP TF. The result shows that, if the OPGS was adopted and the backside ICP etching was done, a $69.3 \%$ (from 5.66 to 9.58 ) and a $253.6 \%$ (from 3.79 to 13.4 ) increase in $\mathrm{Q}_{1}$ was achieved at 5 and $8 \mathrm{GHz}$, respectively. The reason why ICP TF and OPGS-ICP TF exhibit higher $\mathrm{Q}_{1}$ than that of STD TF is mainly attributed to the reduction of the silicon-substrate loss. In addition, in the frequency range of roughly 3-7 GHz, OPGS-ICP TF exhibited $\mathrm{Q}_{1}$, which was a little lower than that of ICP TF because there was an additional induced-eddy-current loss in the OPGS.

Fig. 4(b) shows the measured $k_{\operatorname{Im}}$ and $k_{\mathrm{Re}}$ versus frequency characteristics of STD TF, ICP TF, and OPGS-ICP TF. The results show that, if the OPGS was adopted and the backside ICP etching was done, a $10.5 \%$ (from 0.713 to 0.788 ) and a $14 \%$ (from 0.7 to 0.798 ) increase in $k_{\mathrm{Im}}$ was achieved at 5 and $8 \mathrm{GHz}$, respectively. The reason why OPGS-ICP TF exhibited higher $k_{\operatorname{Im}}(0.788$ at $5 \mathrm{GHz})$ than those of STD TF $(0.713$ at $5 \mathrm{GHz})$ and $\operatorname{ICP}$ TF $(0.688$ at $5 \mathrm{GHz})$ was mainly attributed to the better confinement of the magnetic field achieved by the OPGS. Besides, the reason why STD TF exhibited higher
$k_{\mathrm{Re}}(0.514$ at $5 \mathrm{GHz})$ than those of ICP TF $(0.217$ at $5 \mathrm{GHz})$ and OPGS-ICP TF $(0.278$ at $5 \mathrm{GHz})$ was mainly attributed to the larger resistive coupling through the silicon substrate. Moreover, OPGS-ICP TF exhibited larger $k_{\text {Re }}$ than ICP TF because there was an additional resistive coupling through the OPGS.

Fig. 4(c) shows the measured $G_{\mathrm{A} \text { max }}$ versus frequency characteristics of STD TF, ICP TF, and OPGS-ICP TF. The results show that, if the OPGS was adopted and the backside ICP etching was done, a $17.2 \%$ (from 0.662 to 0.776 ) and a $51.1 \%$ (from 0.55 to 0.831 ) increase in $G_{\mathrm{A} \max }$ was achieved at 5 and $8 \mathrm{GHz}$, respectively. The reason why OPGS-ICP TF exhibited higher $G_{\mathrm{A} \max }$ than those of STD TF was mainly attributed to the increase of $\mathrm{Q}_{1}, \mathrm{Q}_{2}$, and $k_{\mathrm{Im}}$ because of the reduction of the silicon-substrate loss and the increase of the magneticfiled confinement. In addition, the reason why OPGS-ICP TF exhibited higher $G_{\mathrm{A} \text { max }}$ than those of ICP TF was mainly attributed to the increase of $k_{\mathrm{Im}}$ and $k_{\mathrm{Re}}$ because of the increase of the magnetic-filed confinement.

Fig. 4(d) shows the measured $\mathrm{NF}_{\text {min }}$ versus frequency characteristics of STD TF, ICP TF, and OPGS-ICP TF. The results show that, if the OPGS was adopted and the backside ICP etching was done, a $0.682-\mathrm{dB}$ (from 1.795 to $1.113 \mathrm{~dB}$ ) and a $1.79-\mathrm{dB}$ (from 2.597 to 0.807 ) reduction in $\mathrm{NF}_{\min }$ were achieved at 5 and $8 \mathrm{GHz}$, respectively. The reason why OPGS-ICP TF exhibited lower $\mathrm{NF}_{\min }$ than those of STD TF was mainly attributed to the increase of $\mathrm{Q}_{1}, \mathrm{Q}_{2}$, and $k_{\mathrm{Im}}$ because of the reduction of the silicon-substrate loss and the increase of the magnetic-filed confinement. In addition, the reason why OPGSICP TF exhibited lower $\mathrm{NF}_{\min }$ than those of ICP TF was mainly attributed to the increase of $k_{\operatorname{Im}}$ and $k_{\mathrm{Re}}$ because of the increase of the magnetic-filed confinement. 


\section{CONCLUSION}

Compared with the standard transformer, the OPGS-ICP transformer exhibits significant improvement in $Q$-factors $\left(\mathrm{Q}_{1}\right.$ and $\left.\mathrm{Q}_{2}\right)$, magnetic-coupling factor, and $G_{\mathrm{A} \max }$ (or $\mathrm{NF}_{\min }$ ). In addition, compared with the ICP transformer, although the OPGS-ICP transformer exhibits similar $Q$-factor performances, significant improvement in magnetic-coupling factor and $G_{\mathrm{A} \max }$ (or $\mathrm{NF}_{\min }$ ) were achieved. These results demonstrate that the proposed combination of OPGS and backside ICP dry etching is very promising for high-performance RF-IC applications.

\section{REFERENCES}

[1] B. Razavi, RF Microelectronics. Upper Saddle River, NJ: Prentice-Hall PTR, 1998, pp. 39-48.

[2] A. W. L. Ng and H. C. Luong, "A 1-V $17 \mathrm{GHz} 5 \mathrm{~mW}$ quadrature CMOS VCO based on transformer coupling," in Proc. IEEE ISSCC, San Francisco, CA, 2006, pp. 198-199.

[3] D. J. Cassan and J. R. Long, "A 1-V transformer-feedback low-noise amplifier for 5-GHz wireless LAN in $0.18 \mu \mathrm{m}$ CMOS," IEEE J. SolidState Circuits, vol. 38, no. 3, pp. 427-435, Mar. 2003.

[4] K. Kwok and H. C. Luong, "Ultra-low-voltage high-performance CMOS VCOs using transformer feedback," IEEE J. Solid-State Circuits, vol. 40, no. 3, pp. 652-660, Mar. 2005.

[5] C. P. Yue and S. S. Wong, "On-chip spiral inductors with patterned ground shields for Si-based RF IC's," IEEE J. Solid-State Circuits, vol. 33, no. 5, pp. 743-752, May 1998.

[6] K. T. Ng, B. Rejaei, and J. N. Burghartz, "Substrate effects in monolithic RF transformers on silicon," IEEE Trans. Microw. Theory Tech., vol. 50, no. 1, pp. 377-383, Jan. 2002.

[7] K. Chong and Y. H. Xie, "High-performance on-chip transformers," IEEE Electron Device Lett., vol. 26, no. 8, pp. 557-559, Aug. 2005.

[8] M. Ozgur, M. E. Zaghloul, and M. Gaitan, "Optimization of backside micromachined CMOS inductors for RF applications," in Proc. IEEE Int. Symp. Circuits Syst., Geneva, Switzerland, May 2000, pp. V-185-V-188.

[9] H. W. Chiu, S. S. Lu, and Y. S. Lin, "A $2.17 \mathrm{~dB}$ NF, $5 \mathrm{GHz}$ band monolithic CMOS LNA with $10 \mathrm{~mW}$ DC power consumption on a thin $(20 \mu \mathrm{m})$ substrate," IEEE Trans. Microw. Theory Tech., vol. 53, no. 3, pp. 813824, Mar. 2005.

[10] T. H. Lee, The Design of CMOS Radio Frequency Integrated Circuits, 2nd ed. Cambridge, U.K.: Cambridge Univ. Press, 2004, pp. $148-158$.

\section{Simulation Results for Nanoscale Field Effect Diode}

Iraj Sheikhian and Farshid Raissi

\begin{abstract}
Using the PISCES-IIb semiconductor drift-diffusion solver, we have simulated the current-voltage $(I-V)$ curve of a nanoscale fieldeffect diode (FED) with a gate length of $75 \mathrm{~nm}$ and a gate oxide thickness of $2 \mathrm{~nm}$. Whereas devices longer than $100 \mathrm{~nm}$ provide $I_{\mathrm{ON}} / I_{\mathrm{OFF}}$ ratios that are larger than those of comparable silicon-on-insulator MOSFETs (SOI-MOSFETs), the 75-nm device provides a very poor $I_{\mathrm{ON}} / I_{\mathrm{OFF}}$ ratio. A modified version of an FED is proposed, which provides an $I_{\mathrm{ON}} / I_{\mathrm{OFF}}$ ratio that is an order of magnitude larger than that of a comparable SOI-MOSFET for portions of its $I-V$ curve. It appears that the FED can be considered as a viable structure for some digital circuits at these device lengths, provided that minor modifications are made to the regular CMOS process.
\end{abstract}

Index Terms-Field effect diode (FED), nanoelectronics, nanotechnology, nMOSFET.

\section{INTRODUCTION}

Due to problems encountered with regular MOSFETs at device lengths smaller than $80 \mathrm{~nm} \mathrm{[1]-[6],} \mathrm{chief} \mathrm{among} \mathrm{them} \mathrm{being} \mathrm{the}$ problems caused by decreasing the thickness of the gate oxide layer to obtain a large $I_{\mathrm{ON}} / I_{\mathrm{OFF}}$ ratio, it would be constructive to look for alternative structures that could provide larger $I_{\mathrm{ON}} / I_{\mathrm{OFF}}$ ratios. It has been shown that a field effect diode (FED) [7] provides a current in its ON state that is up to two orders of magnitude larger than that of a comparable silicon-on-insulator MOSFET (SOI-MOSFET) for device lengths longer than $200 \mathrm{~nm}$ [8]. At these device lengths, digital and analog circuits can benefit with regards to speed and power consumption if FED devices are used in their design in place of MOSFETs [8], [9].

In this brief, we present simulation results for a nanoscale SOIFED. Interestingly, simulations show that the previously proposed FED design does not seem to provide improvements over a regular MOSFET at device lengths shorter than $100 \mathrm{~nm}$. At such small lengths, injection of excess minority carriers from the source and the drain into the channel interferes with the normal operation of the device, and it does not turn off. However, a simple change in the doping profiles of the source and the drain results in a new structure that is capable of providing much improvement in the $I_{\mathrm{ON}} / I_{\mathrm{OFF}}$ ratio compared to an SOI-MOSFET with the same dimensions. These simulations demonstrate the advantages of an FED over a regular MOSFET at nanoscale dimensions regarding its current-handling capability and its smaller turn-off current, but, as will be shown, modifications are needed in the CMOS process to fabricate the FED.

\section{FED}

An FED is basically a regular SOI-MOSFET structure with two gates over its channel [7]. These gates can be biased such that either a $\mathrm{p}-\mathrm{n}$ or $\mathrm{n}-\mathrm{p}$ channel replaces the lightly doped or intrinsic region between the source and the drain. When a voltage is applied from the

Manuscript received August 24 2006; revised November 10, 2006. The review of this brief was arranged by Editor C. Y. Lu.

The authors are with the Department of Electrical Engineering, Izeh University and the Department of Electrical Engineering, K. N. Toosi University of Technology, Tehran 16314, Iran (e-mail: raissi@kntu.ac.ir).

Color versions of one or more of the figures in this brief are available online at http://ieeexplore.ieee.org.

Digital Object Identifier 10.1109/TED.2006.890600 